\title{
Editorial
}

\section{Metamaterials and Smart Structures in a Big Data Era}

\author{
Ying Wang, ${ }^{1}$ Francesco Dell'Isola, ${ }^{2}$ Tiejun Liu, ${ }^{3}$ and Chunhui Yang ${ }^{4}$ \\ ${ }^{1}$ Department of Civil and Environmental Engineering, University of Surrey, Guildford, Surrey, UK \\ ${ }^{2}$ Faculty of Engineering, University of Rome "La Sapienza", Rome, Italy \\ ${ }^{3}$ School of Civil and Environment Engineering, Urban \& Civil Engineering Disaster Prevention \& Reduction Research Center, \\ Shenzhen Graduate School of Harbin Institute of Technology, Shenzhen, Guangdong, China \\ ${ }^{4}$ School of Computing, Engineering and Mathematics, Western Sydney University, Sydney, NSW, Australia \\ Correspondence should be addressed to Ying Wang; ying.wang@surrey.ac.uk
}

Received 18 December 2016; Accepted 19 December 2016; Published 8 February 2017

Copyright (C) 2017 Ying Wang et al. This is an open access article distributed under the Creative Commons Attribution License, which permits unrestricted use, distribution, and reproduction in any medium, provided the original work is properly cited.

Nowadays, there is no doubt that we have been into the big data era. Under such a circumstance, developing future smart materials and structural systems is largely dependent on big data analysis. This offers not only opportunities to help engineers improve the safety and maintainability of critical structures but also new challenges which required further advances in fundamental research and applied technologies. On the other hand, wide technical possibilities are opened by the design of new metamaterials in sound and vibration control, biomechanics, nanotechnology, and extreme mechanics.

Based on the above considerations, we gathered together in 2016 and proposed this special issue which is aimed at serving a research milestone that summarizes the most recent progress in the field of metamaterials and smart structures.

To ensure the paper quality, rigorous reviews have been performed, and the final acceptance rate is around $40 \%$. The 12 papers appearing in this special issue address a wealth of topics relevant to the proposed topics. This special issue discusses various materials including different characteristics and performances of $1 \mathrm{D}$ photonic crystals and the effect of nanosilica on the fresh properties of cement-based grouting material. It also discusses different structural damage detection methods, including musical tone law method by using vibration data, delamination detection based on laser ultrasonic imaging, bridge deflection monitoring using CCD cameras, and quick structural monitoring and damage assessment technique using smartphone. Further, it addresses the finite element computations of contact problems in micropolar elasticity, as well as vibration and acoustic emission tests on scaled structural models.

\section{Acknowledgments}

As the gust editors of this special issue, we would like to thank the authors who willingly submitted their works of high quality to bring about this special issue. Likewise, the great help of the reviewers is also appreciated, and their careful observations, valuable comments, and suggestions contributed significantly to improve the content of this special issue. Finally, we would like to express our sincere gratitude to the editors of this journal, for encouraging and agreeing to this special issue, which was indispensable in putting together this special issue.

Ying Wang

Francesco Dell'Isola

Tiejun Liu

Chunhui Yang 

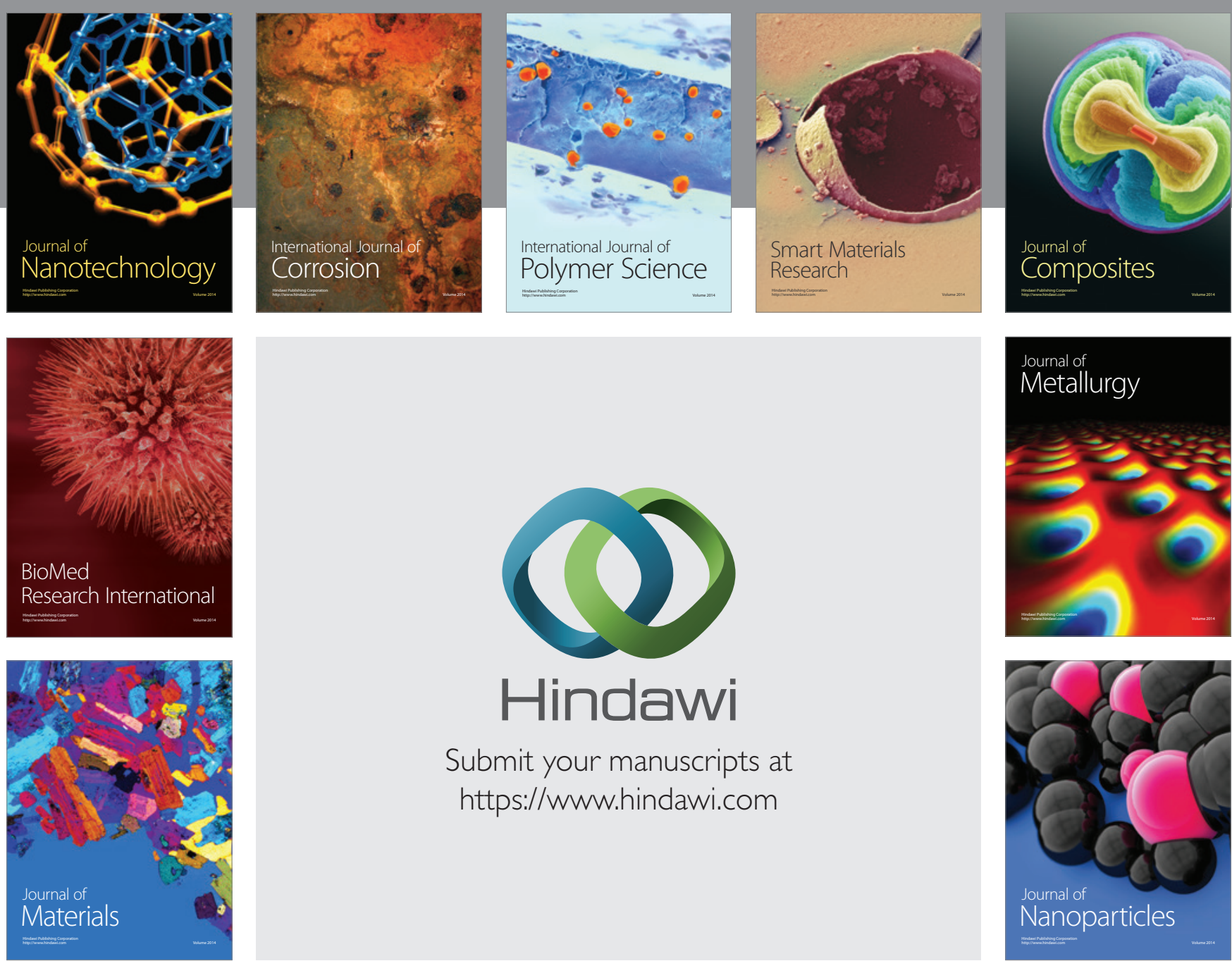

\section{Hindawi}

Submit your manuscripts at

https://www.hindawi.com

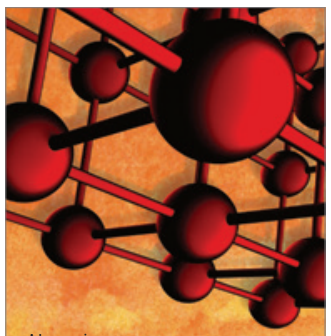

Materials Science and Engineering
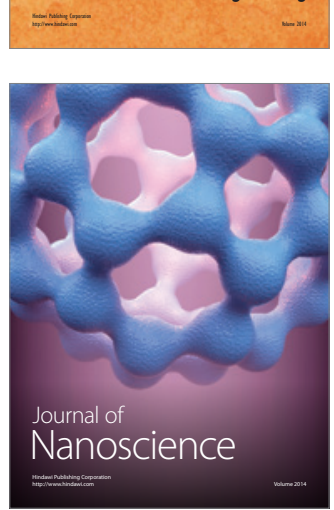
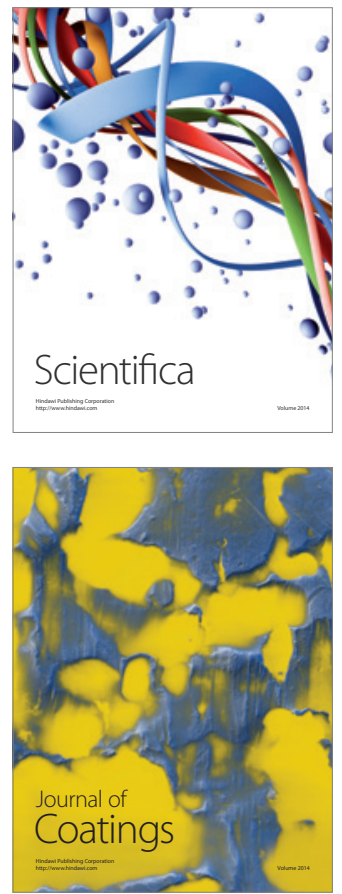
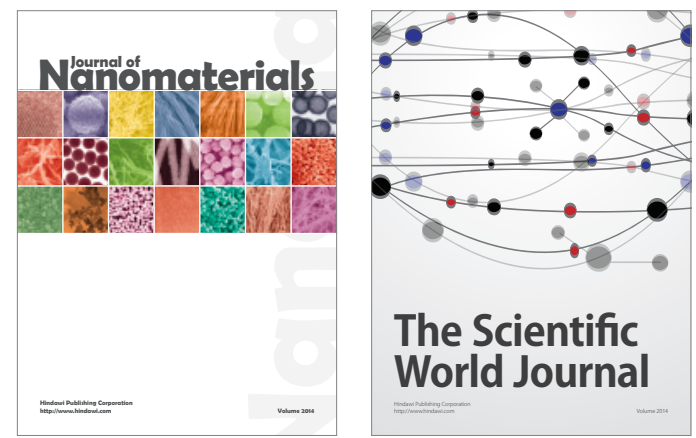

The Scientific World Journal
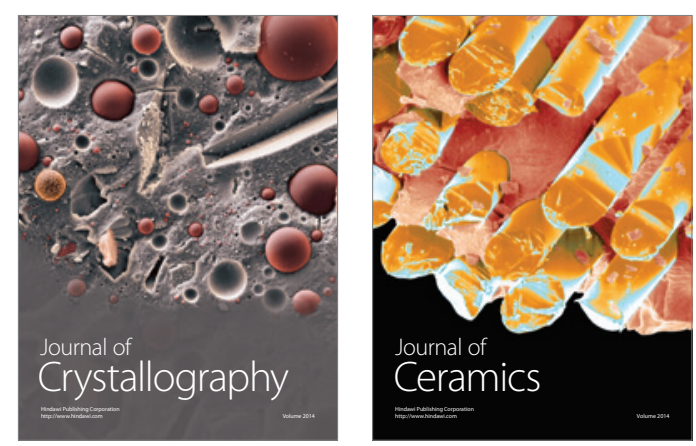
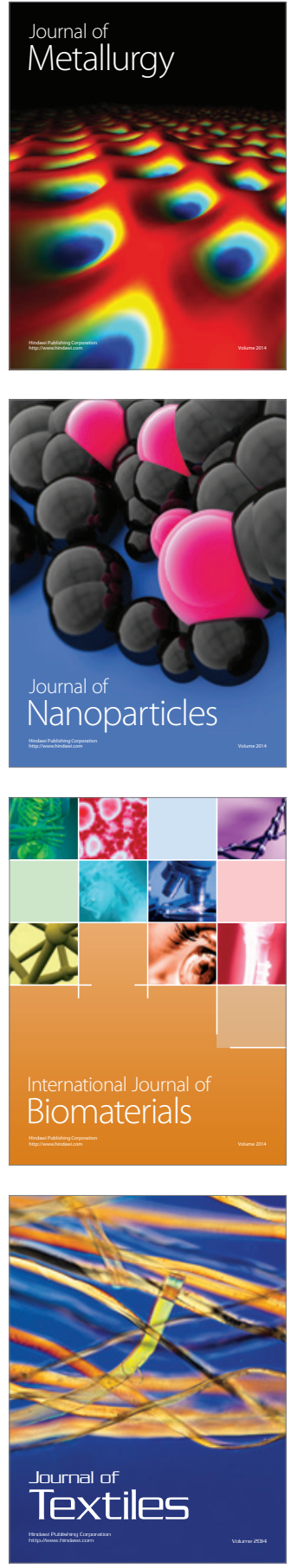